\title{
Aplicación práctica del aprendizaje basado en proyectos en los estudios de Ingeniería Naval y Oceánica
}

\begin{abstract}
Carlos Mascaraque Ramíreza , Lorena Para González ${ }^{\mathrm{b}}$, Jerónimo Esteve Pérez ${ }^{\mathfrak{c}}$, José Enrique Gutiérrez Romero ${ }^{d}$, Juan Francisco Muñoz Rosas ${ }^{\mathrm{e}}$ y Encarnación Álvarez Verdejo ${ }^{\mathrm{f}}$

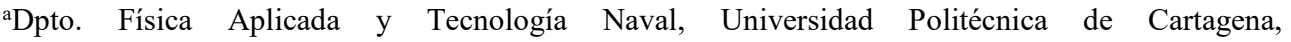
carlos.mascaraque@upct.es, ${ }^{b}$ Dpto. Ciencias Jurídicas y Económicas, Universidad Isabel I, lorena.para@uil.es, 'Dpto. Física Aplicada y Tecnología Naval, Universidad Politécnica de Cartagena, jeronimo.esteve@upct.es, Dpto. Física Aplicada y Tecnología Naval, Universidad Politécnica de Cartagena, jose.gutierrez@upct.es, ${ }^{e} D$ pto. Métodos Cuantitativos para la Economía y la Empresa, Universidad de Granada, jfmunoz@ugr.es, fDpto. Métodos Cuantitativos para la Economía y la Empresa, Universidad de Granada, encarniav@ugr.es.
\end{abstract}

\begin{abstract}
The Project Based Learning (PBA) methodology has been identified as one of the most suitable for improving the competences of students from different areas of knowledge and level of studies. This research focuses on the implementation of this technique in the Master's studies in Naval and Oceanic Engineering, analysing its suitability for this academic level, identifying the most optimal guidelines for its execution in lessons and being able to measure the initial expectations that students have with the use of this teaching methodology.

This work will reflect that the use of the PBA is more than suitable for the technical studies of any branch of engineering, it will identify that following a few simple initial steps this technique can be implemented in any subject, and it will show that the initial results obtained reflect a high level of expectation on the part of the students, who hope to consolidate competences as relevant as teamwork, self-learning skills or technical knowledge, as well as to help them both, in their present academic and in their professional future.
\end{abstract}

Keywords: Project Based Learning, practical application, university education, engineering studies.

\section{Resumen}

En los últimos años, la metodología de Aprendizaje Basado en Proyectos $(A B P)$ se ha identificado como una de las más adecuadas para mejorar las competencias de los estudiantes de diferentes áreas de conocimientos y nivel de estudios. Esta investigación se centra en la implementación de dicha técnica en los estudios de Máster en Ingeniería Naval y Oceánica, analizando su idoneidad para éste nivel académico, identificar las pautas 
más óptimas para su ejecución en las clases y poder medir las expectativas iniciales que tienen los estudiantes con el uso de esta metodología docente.

Este trabajo reflejará que el uso del ABP es más que idóneo para los estudios técnicos de cualquier rama de la ingeniería, identificará que siguiendo unos sencillos pasos iniciales se puede implementar esta técnica en cualquier asignatura, y mostrará que los resultados iniciales obtenidos reflejan un alto nivel de expectación por parte del alumnado, el cual espera consolidar competencias tan relevantes como el trabajo en equipo, las habilidades de autoaprendizaje o los conocimientos técnicos, así como ayudarles tanto en su presente académico, como en su futuro profesional.

Palabras clave: aprendizaje basado en proyectos, aplicación práctica, docencia universitaria, estudios de ingeniería.

\section{Introducción}

\subsection{Descripción del Aprendizaje Basado en Proyectos}

El Aprendizaje Basado en Proyectos (ABP) constituye un marco idóneo para desarrollar las competencias clave y generar experiencias memorables de aprendizaje, en el que el alumnado aprende haciendo (LarmerMergendoller y Boss, 2015). El ABP es una metodología activa, que poder ser completada con otras, como, por ejemplo, aprendizaje servicio, aprendizaje cooperativo, gamificación y/o aula invertida. El ABP sitúa a los alumnos en el centro del proceso de aprendizaje gracias a un planteamiento mucho más motivador en el que entran en juego el intercambio de ideas, la creatividad y la colaboración.

Según Markham (2003) el Aprendizaje Basado en Proyectos (ABP) se puede definir de la siguiente manera: "PBL integrates knowing and doing. Students learn knowledge and elements of the core curriculum, but also apply what they know to solve authentic problems and produce results that matter" PBL integra saber y hacer. Los estudiantes aprenden conocimientos y elementos del plan de estudios principal, pero también aplican lo que saben para resolver problemas auténticos y producir resultados importantes). En este sentido, es obligatorio mencionar la figura de John Dewey, quien generó un impacto único en la educación (Dewey, 1910). De hecho, a partir de conceptos originales introducidos por Dewey, quien también promovió la idea de aprender haciendo, la investigación educativa avanzó en esta idea de enseñar y aprender dando lugar a la metodología definida antes.

En el ABP se pueden distinguir las siguientes fases:

- Punto de partida (formulación de la pregunta inicial).

- Formación de equipos colaborativos.

- Definición del reto final.

- Organización y planificación.

- Búsqueda y recopilación de información.

(c)) BY-NC-ND 2019, Universitat Politècnica de València 
- Análisis y síntesis.

- Producción.

- Presentación del proyecto.

- Respuesta colectiva a la pregunta inicial.

- Evaluación y autoevaluación.

- Aprendizaje significativo.

Baje estas premisas, algunas investigaciones llevadas a cabo en lso últimos años, como la de Pérez (2008), han identificado la idoneidad del ABP para la mejora de la enseñanza a nuivel universitario, siendo perfectamente válidad en las titulaciones técnicas superiores (Cabedo et al., 2017), por lo tanto es extrapolable a los estudios de Máster en Ingeniería Naval y Oceánica.

Para optimizar este tipo de metodología, debe adaptarse al nivel de estudios elegido, y por lo tanto en el caso analñizado a los niveles de máster en ingeniería (García et al., 2016). Atendiendo no solo al nivel de estudios, debiendo centrarse tambie'n en el ámbito de conocimiento de la trama de ingeniería impartida.

Una de las ventajas que también ofrece esta metodología, es la posibilidad de combinarla a otras técnicas docentes, como las dinámicas de grupo (VelascoScott y Aguado, 2017), las Tecnologías de Información y Comunicación (TIC) (Ausín et al., 2016), u otras técnicas de aprendizaje colaboratico (RodriguezHudson y Niblock, 2018).

\subsection{Descripción de los títulos de Ingeniería Naval y Oceánica}

El título de Máster en Ingeniería Naval y Oceánica se recoge en el mapa de nuevas titulaciones de Grado y Máster, y su adscripción a los Centros de la Universidad Politécnica de Cartagena al amparo del Real Decreto 1393/2007, de 29 de octubre, de ordenación de las enseñanzas universitarias oficiales y al Real Decreto RD/861/2010, de 2 de julio, que modifica al anterior, así como a lo indicado en la Orden Ministerial CIN/354/2009, de 9 de febrero, por la que se establecen los requisitos para la verificación de los títulos universitarios oficiales que habiliten para el ejercicio de la profesión de Ingeniero Naval y Oceánico (ETSINO, 2019)

Se trata de un título de Máster con orientación profesional con las competencias que se establecen en la citada Orden Ministerial, con el objetivo de garantizar la formación adecuada en las actividades tecnológicas ligadas al ámbito de la ingeniería naval (proyecto, ingeniería de fabricación, dirección de obra, inspección técnica, seguridad, salvamento y rescate, apoyo logístico, mantenimiento, transformaciones, reformas, reparaciones, etc.) y que se desarrollan, principalmente, sobre los siguientes campos tecnológicos:

- Buques y embarcaciones de todo tipo.

- Plataformas y artefactos flotantes y fijos (diques flotantes, prospección y aprovechamiento de recursos marítimos, etc.).

- Acuicultura y sistemas de pesca.

- Gestión de empresas marítimas (astilleros, navieras, etc.). 
Las atribuciones profesionales relacionadas con estos campos tecnológicos son cubiertas en su totalidad por el Ingeniero Naval y, posteriormente, por el Ingeniero Naval y Oceánico, que sustituyó como título oficial al anterior ampliando sus competencias.

\section{Objetivos}

Los objetivo principal de esta investigación es mejorar el aprendizaje y asimilación de conceptos por parte de los alumnos del Máster en Ingeniería Naval y Oceánica, para ello se va a proceder a la aplicación de la metodología de Aprendizaje Basado en Proyectos en una de las asignaturas de la titulación, esperando validar el empleo de esta metodología, así como identificar las necesidades para su correcta implementación y las posibles dificultades que pueden surgir en el proceso.

\section{Desarrollo de la innovación}

El proceso para la implementación de la metodología $\mathrm{ABP}$ en la titulación de Ingeniería Naval y Oceánica comienza con la elección de la asignatura donde se aplicará, en este caso se ha elegido la asignatura de Ingeniería de Sistemas Aplicada. Esta asignatura se caracteriza por ser obligatoria, de 6 créditos, con una carga total de 180 horas para el alumnado, localizadas en el segundo cuatrimestre del primer curso del máster. Investigaciones previas, como el de Rico-JiménezGaray-Jiménez y Ruiz-Ledesma (2018), han verificado la adecuación de este tipo de metodología en estudios de ingeniería aplicada.

La elección de dicha asignatura se debe principalmente al contenido de su temario, donde se trata de la filosofía de la Ingeniería de Sistemas en el sector naval, lo que puede resumirse como el estudio de las diferentes fases del proceso de diseño y construcción de un buque, y la toma de decisiones sobre el alcance de los trabajos a desarrollar en cada una de dichas fases, así como su control y monitorización.

Una vez seleccionada la asignatura, el siguiente paso es decidir el proyecto a desarrollar a lo largo de las clases (Martín y Martínez, 2018), es recomendable que dicho proyecto sea de interés para el alumnado, por lo tanto que sea actual y vinculado con los estudios cursados por lo estudiantes, y al mismo tiempo debe tener una complejidad acorde al nivel del curso en el que se encuentran los estudiantes. Bajo estas premisas, se identifica las opciones de realizar una fragata, una corbeta o un patrullero, debido a la alta complejidad de la fragata esta opción es descartada, y por tratarse de un tema más actual, España está en proceso de construcción y entrega de varios Patrulleros Oceánicos, este es el buque que finalmente se elige.

En los siguientes apartados se describirá brevemente el proyecto a desarrollar, así como la metodología a seguir en el aula. 


\subsection{Descripción del proyecto a desarrollar: Buque Patrullero Oceánico}

Un buque Patrullero Oceánico se concibe para llevar a cabo misiones de control del mar en escenarios de baja intensidad, con capacidad de disuasión y reacción ante amenazas asimétricas o convencionales de pequeña entidad.

Se trata de un buque de tamaño moderado, de una eslora total de entre 80-100 m y un desplazamiento, dependiendo del tipo específico de patrullero, comprendido entre $1.500 \mathrm{y}$ 2.700 t. Asimismo, tiene una dotación reducida (55-100 personas), alto nivel de habitabilidad, dotado de sistemas de estabilización activos y pasivos, con capacidad para la navegación en mares adversos lo que le permite realizar adecuadamente las misiones asignadas dentro del ámbito oceánico.

Este tipo de buques dispone de una gran autonomía, que le dota de un amplio radio de acción de aproximadamente 3.500 millas náuticas, y una velocidad moderada/alta, en torno a 25 nudos, que le permite acceder a la zona de operación en un tiempo reducido.

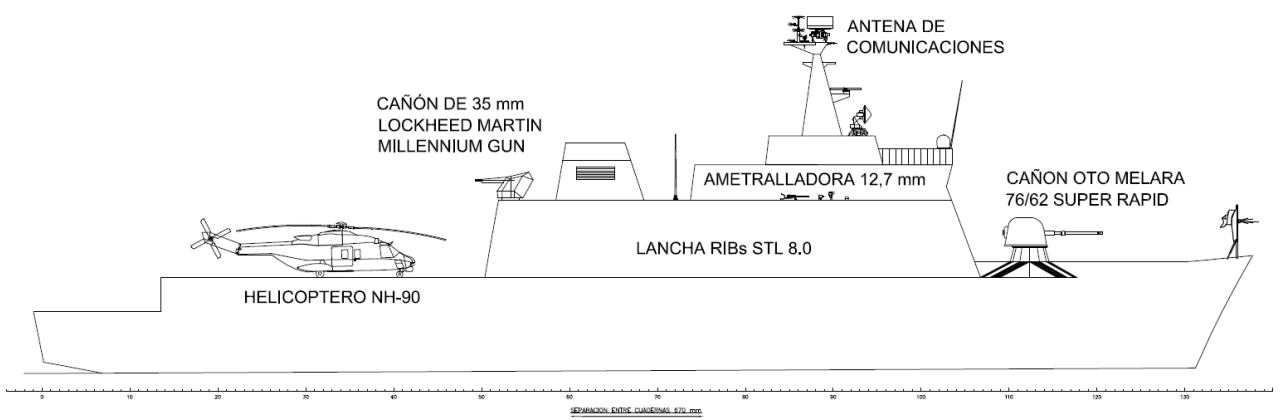

ф.

Fig. 1 Ejemplo de Patrullero Oceánico. Fuente: Elaboración propia

Estos buques disponen de una cubierta de vuelo apta para operar con helicópteros de tamaño medio y un hangar fijo, lo que proporciona una gran versatilidad en las diferentes misiones previstas (evacuación, rescate, lucha contra el narcotráfico, etc.), pudiendo llevar a cabo, además, operaciones visuales de vuelo diurnas y nocturnas y aprovisionamiento en la mar de sólidos ligeros y líquidos.

Asimismo, también pueden disponer de una zona hospitalaria que proporciona apoyo médico de primeros auxilios y estabilización para posterior evacuación médica, así como, de telemedicina con el objeto de potenciar las posibilidades de atención médica.

Una característica relevante del diseño es el alto estándar de habitabilidad tanto para la dotación básica como para el personal de transporte, incorporando los medios más actuales en cuanto a confort, control medioambiental y diseño ergonómico, de modo que se faciliten las condiciones de vida a bordo, permitiendo la operación del buque en lugares alejados de las bases durante periodos de tiempo prolongados.

Otro aspecto importante del diseño es la flexibilidad ofrecida por la plataforma al tener la posibilidad de incrementar su capacidad en determinadas funciones mediante la estiba en la 
cubierta de trabajo y/o de vuelo de contenedores que pueden incorporar distintos equipos/sistemas, tales como sistemas de lucha contra la contaminación, contenedores vida, contenedores con equipos de Vehículos Autónomos Submarinos, etc., lo que permite optimizar el uso de la plataforma dado que determinados equipos y/o sistemas solo se embarcan a bordo cuando son necesarios para el perfil de misión previsto.
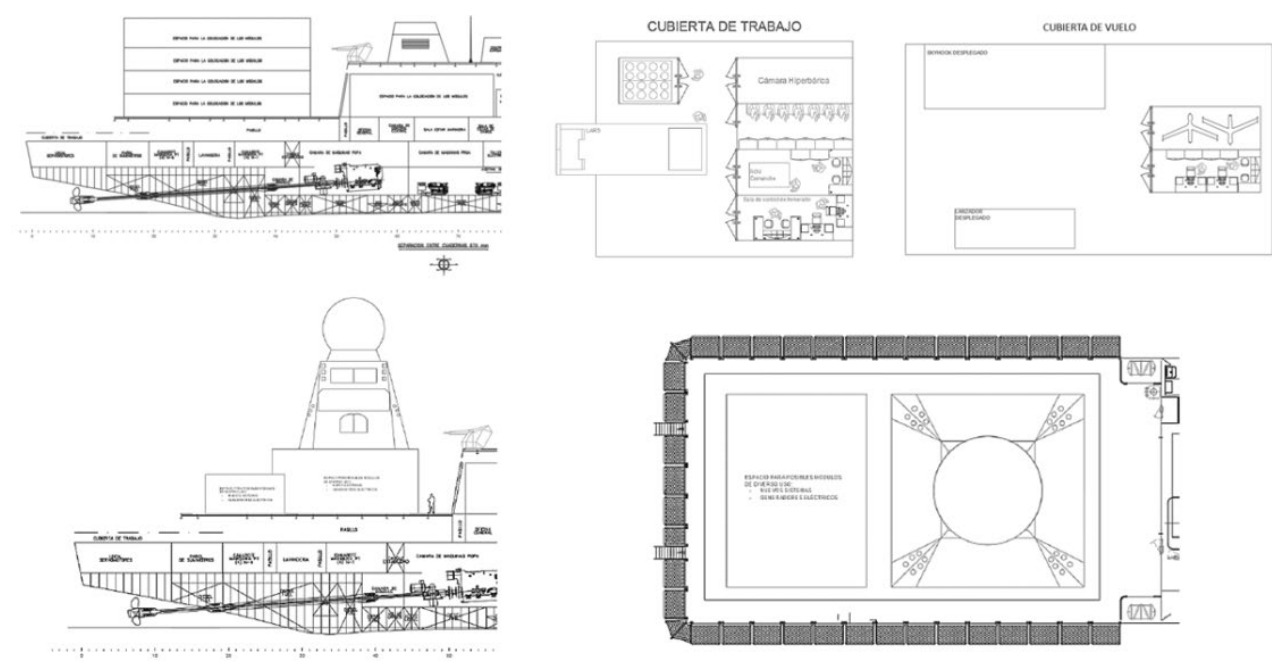

Fig. 2 Ejemplo de diferentes configuraciones en plataforma multipropósito. Fuente: Elaboración propia

Estos buques están orientados a tareas de vigilancia y patrulla con un alto grado de versatilidad en la plataforma, lo que da como resultado un buque de altas prestaciones, no solo en el ámbito militar sino también en el de cooperación en tareas de salvamento, lucha contra la contaminación, evacuación, ayuda humanitaria, etc. (Ingeniería-Naval, 2011). De forma no exhaustiva las misiones de este buque son:

- Operaciones de Control de Mar en Escenarios de Baja Intensidad.

- Presencia Naval.

- Protección y Escolta.

- Ayuda Humanitaria.

- Apoyo a Desastres.

- Rescate y Salvamento.

- Operaciones contra el Narcotráfico.

- Operaciones contra el Tráfico de Personas.

- Vigilancia y control de legislación medioambiental.

- Apoyo en operaciones de lucha anti-contaminación.

- Apoyo logístico y sanitario limitado a buques menores. 
En cuanto a los principales aspectos tecnológicos destacan.

- Alto Grado de Flexibilidad y Polivalencia de la Plataforma.

- Embarque de Sistemas en Contenedores.

- Alto nivel de Automatización compatible con dotación reducida.

- Planta Propulsora Combinada Diésel y Eléctrica.

- Diseño orientado a facilitar el mantenimiento.

- Alto nivel de habitabilidad y seguridad en las operaciones.

- Buque ecológico y funciones de lucha anticontaminación.

\subsection{Metodología a emplear a lo largo del curso académico}

La asignatura consta de 4 horas de docencia presencial a la semana, 2 horas los lunes y 2 horas los miércoles. Las clases de los lunes se emplearán para profundizar en los temas teóricos que componen el temario, y las dos horas de los miércoles para la ejecución del proyecto. Esto implica que cada miércoles los alumnos avanzarán en los distintos puntos que conforman el proyecto, habiendo sido explicados previamente en la clase teórica que se imparte a principios de semana.

Con esto se desarrolla la siguiente planificación para las clases de desarrollo del proyecto:

- Caso Práctico 1: Desarrollo de la estrategia inicial para acometer un proyecto naval de nueva construcción.

- Caso Práctico 2: Definición de procedimientos de control de requisitos, plazo y costes.

- Caso Práctico 3: Definición de un grupo de trabajo en Ingeniería de Sistemas.

- Primera presentación, exposición del estado actual del proyecto.

- Caso Práctico 4: Definición de hitos y niveles TRL de los sistemas principales del buque.

- Caso Práctico 5: Desarrollo de una planificación básica del proyecto.

- Caso Práctico 6: Gestión del coste y el plazo por las técnicas del Valor Ganado (EVMS - ES) del proyecto.

- Caso Práctico 7: Plan de Gestión de Riesgos.

- Presentación final y entrega de la memoria del proyecto.

Como se puede ver, se identifican 7 sesiones de trabajo, 2 sesiones de presentaciones, una para explicar el estado de evolución del proyecto y otra final donde se expondrán los resultados generados por los alumnos. En la sesión correspondiente a la presentación final, lo alumnos entregarán una memoria donde se recogerá todo el trabajo realizado.

Los alumnos formarán equipos de trabajo, formados por un número de entre 4 y 6 componentes, manteniéndose los mismos grupos a lo largo de toda la asignatura.

La ejecución de las clases correspondientes a las sesiones prácticas seguirá un programa como el expuesto en la Tabla 1. 
Tabla 1. Programación tipo para una sesión práctica

\begin{tabular}{|c|c|c|c|c|}
\hline ID & Tarea & Tiempo & Profesor & Estudiantes \\
\hline 1 & $\begin{array}{l}\text { Presentar el ejercicio y los } \\
\text { objetivos que persigue }\end{array}$ & 15 mins & $\begin{array}{c}\text { Realizar } \\
\text { presentación }\end{array}$ & Atender \\
\hline 2 & $\begin{array}{l}\text { En grupo trabajar sobre el caso } \\
\text { presentado }\end{array}$ & $60-70$ mins & $\begin{array}{c}\text { Apoyar y resolver } \\
\text { dudas }\end{array}$ & Desarrollar el caso \\
\hline 3 & $\begin{array}{l}\text { Completar el informe de la } \\
\text { práctica }\end{array}$ & $\begin{array}{l}\text { En paralelo al } \\
\text { punto anterior }\end{array}$ & $\begin{array}{c}\text { Apoyar y resolver } \\
\text { dudas }\end{array}$ & Completar informe \\
\hline 4 & Presentación oral & 3 minutos / grupo & $\begin{array}{l}\text { Atender y realizar } \\
\text { preguntas }\end{array}$ & Exponer / responder \\
\hline 5 & $\begin{array}{l}\text { Feedback del profesor a las } \\
\text { presentaciones }\end{array}$ & 5 a 10 minutos & $\begin{array}{c}\text { Aportar un feedback } \\
\text { de cara a la memoria } \\
\text { y continuidad del } \\
\text { proyecto }\end{array}$ & $\begin{array}{c}\text { Atender y tomar } \\
\text { anotaciones sobre el } \\
\text { feedbak }\end{array}$ \\
\hline
\end{tabular}

\section{Resultados}

$\mathrm{Al}$ estar en curso la asignatura donde se ha realizado la aplicación de la metodología ABP, no se disponen de resultados finales, si bien se ha realizado una encuesta inicial a los alumnos, enfocando las preguntas de dicho cuestionario a las expectativas que tienen de la asignatura y en la metodología del Aprendizaje Basado en Proyectos.

La encuesta pedía a los alumnos, siempre de forma voluntaria, que valoraran en una escala Likert de 1 a 5 , siendo 1 "muy bajas" y 5 "muy altas", las siguientes cuestiones:

- Q1 - Sus expectativas académicas con respecto a la asignatura son...

- Q2 - Sus expectativas con respecto a la asignatura y a cómo esta puede ayudarle o influirle en su futuro profesional son...

- Q3 - Creo que el ABP podría fomentar mi comprensión de unos conocimientos que me brinden la oportunidad de ser original en el desarrollo y/o aplicación de ideas...

- Q4 - Considero que el ABP podría ayudarme a desarrollar habilidades de aprendizaje que me permitan continuar estudiando de un modo auto-dirigido o autónomo...

- Q5 - Pienso que el ABP podría tener utilidad para ayudarme a desarrollar soluciones técnicas, económicas y ambientalmente adecuadas a necesidades de la Ingeniería Naval y Oceánica...

- Q6 - Considero que el ABP podría tener utilidad para fomentar mi capacidad de integración de sistemas marítimos complejos y traducirlos en soluciones viables...

- $\quad$ Q7 - Pienso que el ABP puede fomentar el trabajo en equipo... 
- $\mathrm{Q} 8$ - Considero que el ABP puede ayudarme a utilizar diferentes recursos y fuentes de información...

- $\quad$ Q9 - En general, piensa que el ABP puede ser de ayuda a nivel académico para mi aprendizaje...

- Q10 - En general, piensa que el ABP puede ser de ayuda a nivel profesional...

Con ello se han obtenido los datos que se muestran en la Tabla 2:

Tabla 2. Resultados obtenidos de las encuestas a los alumnos

\begin{tabular}{ccccccccc}
\hline ID & Mínimo & Media & $\begin{array}{c}\text { Desviación } \\
\text { típica }\end{array}$ & $\mathbf{1}$ & $\mathbf{2}$ & $\mathbf{3}$ & $\mathbf{4}$ & $\mathbf{5}$ \\
\hline Q1 & 3 & 3.86 & 0.66 & 0 & 0 & 4 & 8 & 2 \\
Q2 & 3 & 4.07 & 0.47 & 0 & 0 & 1 & 11 & 2 \\
Q3 & 3 & 3.86 & 0.66 & 0 & 0 & 4 & 8 & 2 \\
Q4 & 3 & 4.00 & 0.39 & 0 & 0 & 1 & 12 & 1 \\
Q5 & 2 & 3.71 & 0.83 & 0 & 1 & 4 & 7 & 2 \\
Q6 & 3 & 3.57 & 0.76 & 0 & 0 & 8 & 4 & 2 \\
Q7 & 3 & 4.43 & 0.76 & 0 & 0 & 2 & 4 & 8 \\
Q8 & 3 & 4.07 & 0.62 & 0 & 0 & 2 & 9 & 3 \\
Q9 & 3 & 4.07 & 0.73 & 0 & 0 & 3 & 7 & 4 \\
Q10 & 3 & 4.07 & 0.73 & 0 & 0 & 3 & 7 & 4 \\
\hline TOTAL & $\mathbf{2}$ & $\mathbf{3 . 9 7}$ & $\mathbf{0 . 4 7}$ & $\mathbf{0}$ & $\mathbf{1}$ & $\mathbf{3 2}$ & $\mathbf{7 7}$ & $\mathbf{3 0}$ \\
\hline
\end{tabular}

Gráficamente se representan los resultados en la Figura 3, donde se puede ver que las expectativas del alumnado con la metodología ABP son elevadas, siendo la media de 3.97 en la escala utilizada (de 1 a 5). 


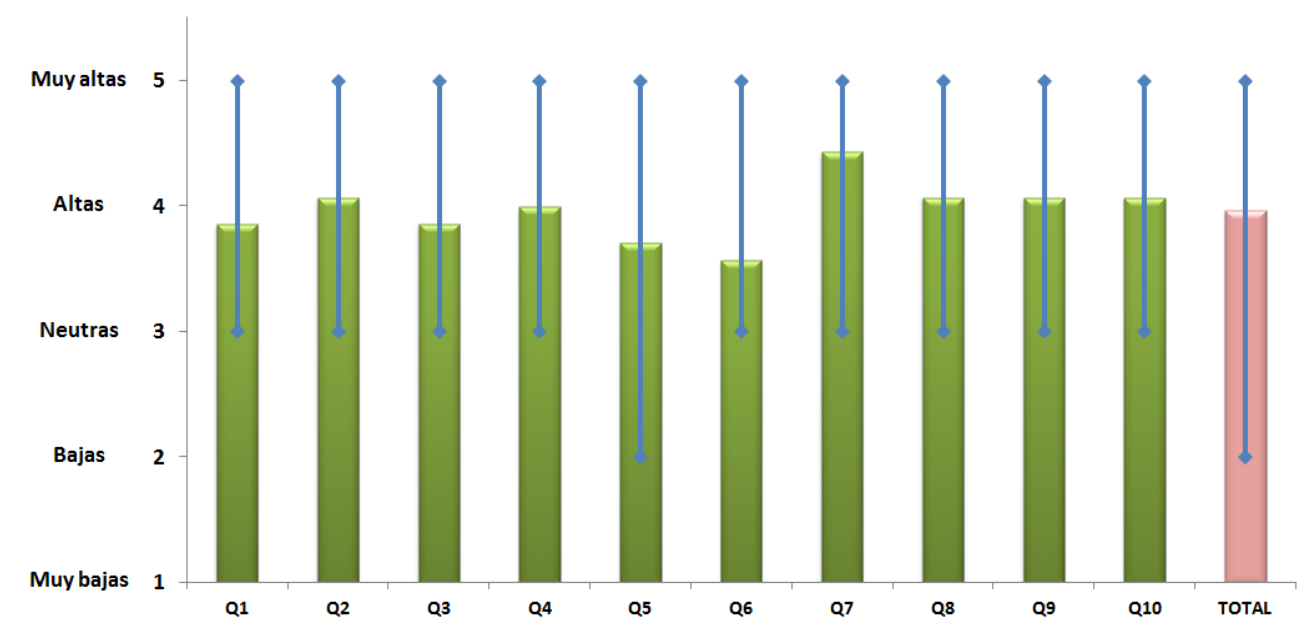

Fig. 3 Exposición gráfica de los resultados obtenidos en las encuestas a los alumnos. Fuente: Elaboración propia

En un análisis de los resultados obtenidos, se desprende una serie de conclusiones iniciales, las cuales pueden ser resumidas en los siguientes puntos de interés:

- La media de las respuestas están siempre por encima de 3, por lo tanto se puede considerar que el alumnado tiene expectativas positivas con el empleo de esta metodología en el aula.

- Todas las cuestiones han obtenido una puntuación máxima de 5, mientras que la mínima ha sido de 3 , a excepción de una única pregunta que ha obtenido un mínimo de 2.

- La pregunta con la media más alta de las respuestas es la relativa al trabajo en equipo, lo cual es coherente con este tipo de metodología docente, y significa que los alumnos han comprendido el modo de proceder en el transcurso de las clases.

- Las preguntas con menor índice de puntuación son las relativas a la consecución de las competencias más técnicas de los conocimientos navales, si bien el resultado es superior a 3.5 puntos. Esto debe interpretarse como el riesgo que ven los alumnos de que esta metodología no profundice suficientemente en los conocimientos más técnicos, con lo que deberá fomentarse estas competencias en el aula, para así hacer ver a los estudiantes que la metodología ABP también mejorará este tipo de competencias.

- La media total de las respuestas es de 3.97 puntos, por lo tanto las expectativas del alumnado son "altas", con lo que se puede considerar una metodología motivadora para el alumnado y por lo tanto de gran interés por parte del profesorado.

\section{Conclusiones}

En los apartados anteriores se ha mostrado el proceso de implementación de la metodología de Aprendizaje Basado en Proyectos (ABP), aplicándose en los estudios de Máster en Ingeniería Naval y Oceánica. Se ha identificado la necesidad de unas pautas iniciales, con el propósito de que la implementación de dicha metodología sea la más idónea y óptima 
posible, lo cual dependerá de la asignatura a impartir, su complejidad, la innovación del proyecto a tratar y la adecuación con la temática de los estudios.

$\mathrm{Al}$ tratarse de un proceso que se encuentra actualmente en desarrollo, no se dispone de datos finales, pero sí se han tomado datos iniciales de las expectativas de los alumnos con la implementación de la metodología ABP en una asignatura obligatoria de 6 créditos de su titulación. Al tratarse de alumnos de primer curso de máster, se considera que tiene suficiente visión crítica para realizar una encuesta inicial de expectativas, debido a su madurez y su veteranía en los estudios universitarios.

Los resultados de las encuestas realizadas reflejan un alto interés por parte de los estudiantes, lo que sin duda es un indicador motivador para el docente a la hora de decantarse por esta metodología, siendo al mismo tiempo una responsabilidad para poder cumplir con las altas expectativas.

Con todo ello puedo concluirse que el $\mathrm{ABP}$ es una metodología idónea para su utilización en los estudios de ingeniería, debiendo atenderse a unas pautas iniciales sencillas y fáciles de seguir, consiguiendo ser motivador para los estudiantes y para los docentes.

\section{Referencias}

AUSÍN, V., ABELlA, V., DELGADO, V. y HORTIGÜELA, D. (2016). "Aprendizaje basado en proyectos a través de las tic: Una experiencia de innovación docente desde las aulas universitarias" en Formación universitaria, vol. 9, issue 3, pp. 31-38.

CABEDO, L., IZQUIERDO, R., ROYO, M., HERNÁNDEZ, L., GIMÉNEZ, I., BELTRÁN, H., MOLINER, L., CABEDO, A., RODA, V. y LAPEÑA, L. (2017). "Experiencias de aprendizajeservicio basado en proyectos en grados de Ingeniería en la Universitat Jaume I" en New competences in Engineering Education in the area of sustainability and university social responsibility, vol., issue, pp. 13-15.

DEWEY, J. (1910). How we think, Boston, MA: D.C. Heath \& Co.

ETSINO (2019). Memoria para la solicitud de verificación del título de máster universitario de ingeniería naval y oceánica por la Universidad Politécnica de Cartagena, Cartagena (Spain): ETSINO (Escuela Técnica Superior de Ingeniería Naval y Oceánica).

GARCÍA, O., CARlos, J., MÁRQUEZ GARCÍA, M. L. y DELGADO OLMOS, Á. H. (2016). "Metodologías innovadoras en la enseñanza y el aprendizaje de la Ingeniería Gráfica" en vol., issue.

INGENIERÍA-NAVAL (2011). "BAM, Buque de Acción Marítima" en Ingeniería Naval, vol. 893, issue, pp. 19-28.

LARMER, J., MERGENDOLLER, J. y BOSS, S. (2015). Setting the standard for project based learning: ASCD.

MARKHAM, T. (2003). Project based learning handbook: A guide to standards-focused project based learning for middle and high school teachers, Oakland, CA: Buck Institute for Education.

MARTÍN, J. G. y MARTÍNEZ, J. E. P. (2018). "Aprendizaje basado en proyectos: método para el diseño de actividades" en Revista Tecnología, Ciencia y Educación, vol., issue 10. 
Aplicación práctica del aprendizaje basado en proyectos en los estudios de Ingeniería Naval y Oceánica

PÉREZ, M. M. (2008). "Aprendizaje basado en proyectos colaborativos. Una experiencia en educación superior" en Laurus, vol. 14, issue 28, pp. 158-180.

RICO-JIMÉNEZ, B. A., GARAY-JIMÉNEZ, L. I. y RUIZ-LEDESMA, E. F. (2018). "Implementación del aprendizaje basado en proyectos como herramienta en asignaturas de ingeniería aplicada/Implementation of project-based learning as a tool in applied engineering subjects" en RIDE Revista Iberoamericana para la Investigación y el Desarrollo Educativo, vol. 9, issue 17, pp. 20-57.

RODRIGUEZ, C., HUDSON, R. y NIBLOCK, C. (2018). "Collaborative learning in architectural education: Benefits of combining conventional studio, virtual design studio and live projects" en British Journal of Educational Technology, vol. 49, issue 3, pp. 337-353.

VELASCO, M. S., SCOTT, S. M. y AGUADO, R. M. (2017). "Evaluación del trabajo de grupo a través de la participación en proyectos de aprendizaje tutorado. Reflexiones y propuestas" en Revista Infancia, Educación y Aprendizaje, vol. 3, issue 2, pp. 94-100. 